\title{
Lipoma de corpo caloso: relato de caso
}

\section{Lipoma corpus callosum: a case report}

\author{
Francisco Daniel Bezerra Amorim ${ }^{1}$. Isabela Orieta de Oliveira Macedo ${ }^{1}$. Moacir Pereira Leite Neto ${ }^{2}$. Francisco \\ Marcos Bezerra da Cunha ${ }^{3}$. Isabel Monique Leite Romualdo ${ }^{4}$
}

1 Residente de Clínica Médica, Universidade Federal do Cariri, Juazeiro do Norte, Ceará, Brasil. 2 Mestrado em Ciências da Saúde, Especialista em Neurologia e Neurofisiologia Clínica, Professor das disciplinas de neurologia e semiologia, Universidade Federal do Cariri, Juazeiro do Norte, Ceará, Brasil. 3 Doutorado em Neurologia, Especialista em Neurologia e Neurofisiologia Clínica, Professor adjunto de Neurologia, Universidade Federal do Cariri, Juazeiro do Norte, Ceará, Brasil. 4 Enfermeira, Especialista em Política em Saúde Coletiva, Universidade Regional do Cariri, Crato, Ceará, Brasil.

\section{RESUMO}

Lipoma de corpo caloso é uma condição clínica rara. A epilepsia é a manifestação mais comum, mas também há casos assintomáticos, sendo um achado ocasional em exames de imagem ou autopsia. O caso relata uma situação clínica infrequente, cujo diagnóstico foi definido após realização de exames de neuroimagem. Paciente de 39 anos, que aos 10 anos de idade apresentou crise convulsiva, sendo iniciado tratamento com fenobarbital, ficou em uso deste por 4 anos com bom controle do quadro, sendo suspenso após esse período, e permanecendo assintomática. No entanto, durante a gravidez, houve recidiva das crises, com retomada do uso das medicações, mesmo assim esta persistiu com dois episódios convulsivos ao mês. A paciente foi submetida a exames de neuroimagem que evidenciaram o lipoma de corpo caloso, sendo optado pelo tratamento clínico. Diante dos avanços nos métodos de neuroimagem para diagnóstico, a probabilidade de essa má-formação ser diagnosticada, mesmo quando assintomática, tem aumentado. A ressecção do lipoma de corpo caloso, como descritos na literatura, tem mostrado resultados catastróficos, sendo assim, em casos assintomáticos ou achado incidental o tratamento deve ser conservador, como no caso da paciente em estudo.

Palavras-chave: Lipoma. Corpo caloso. Epilepsia.

\begin{abstract}
Corpus callosum lipoma is a rare clinical condition. Epilepsy is the most common manifestation but there are also asymptomatic cases, being an occasional finding in imaging or autopsy. The case reports an infrequent clinical situation whose diagnosis was defined after performing neuroimaging exams. The information described in this study was obtained by reviewing the patient's medical records, photographic record of the image exams performed by the patient and review of the literature. Patient of 39 years old, who at 10 years of age had seizures, being initiated treatment with fenobarbital which was in use for 4 years, with good control of the condition, being suspended after this period and remaining asymptomatic. However, during pregnancy, there was recurrence of seizures, with resumption of medication use, and yet persisting with two convulsive episodes a month. The patient underwent neuroimaging tests that evidenced corpus callosum lipoma, having opted for clinical treatment. In face of the advances in neuroimaging methods for diagnosis, the probability of this malformation being diagnosed, even when asymptomatic, has increased. The resection of corpus callosum lipoma, as described in the literature, has shown catastrophic results, thus, in asymptomatic cases or incidental finding, the treatment should be conservative, as in the case of the patient under study.
\end{abstract}

Keywords: Lipoma. Corpus callosum. Epilepsy.

Autor correspondente: Francisco Daniel Bezerra Amorim, Rua Joaquim Nabuco, 1400, Meireles, Fortaleza, Ceará. CEP: $60125-120$. Telefone: +55 85 98677-3602. E-mail: daniel_bamorim@hotmail.com

Conflito de interesses: Não há qualquer conflito de interesses por parte de qualquer um dos autores.

Recebido em: 20 Fev 2018; Revisado em: 29 Jul 2018; Aceito em: 29 Jul 2018. 


\section{INTRODUÇÃO}

O lipoma de corpo caloso ( $\mathrm{LCC}$ ) é descrito como sendo um raro tumor benigno intracraniano, tendo muitos casos descritos somente após a morte, com uma estimativa de incidência de $0,06 \%$ e representando menos de $0,1 \%$ dos tumores intracranianos. ${ }^{1}$ Vonderahe e Niemer descreveram uma ocorrência de 0,08\% após analise de 5000 autópsias de LCC, e uma rara associação com agenesia. ${ }^{2}$ A porção anterior do corpo caloso é o principal sitio de localização topográfica. ${ }^{3}$

A formação embriogênica do corpo caloso dá-se no sentido anterior para posterior, exceto a porção rostral. Más formações congênitas podem ocorrer, e a agenesia do corpo caloso pode ser parcial ou total. No caso de agenesia parcial do corpo caloso tanto o esplênio como o rostro estarão ausentes, sendo que a presença do esplênio normal nos direciona para um processo secundário, sendo denominado de hipoplasia. O lipoma de corpo caloso está associado a graus diferentes de agenesia. Acredita-se que quanto mais precoce for o aparecimento do lipoma, maior será a gravidade da alteração resultante do corpo caloso. ${ }^{4}$

No que tange aos aspectos clínicos, são descritas associações de crises convulsivas e de comprometimento cognitivo. ${ }^{5} \mathrm{~A}$ epilepsia é uma doença caracterizada por crises recorrentes espontâneas resultantes de descargas elétricas paroxísticas descontroladas nos neurônios no cérebro, e que pode ser secundária a várias condições clínicas. ${ }^{6}$ Ultimamente, com os avanços no conhecimento das etiologias das epilepsias, sustentado por avanços dos exames de neuroimagem e testes genéticos, as epilepsias passaram a ser descritas por suas etiologias subjacentes específicas, podendo ser de causa genética, estrutural, metabólica, imune, infecciosa, desconhecida e epilepsias não classificadas. Epilepsia é a apresentação mais comum do lipoma de corpo caloso. ${ }^{7}$

A ressonância magnética (RNM) é o exame mais preciso para o seu diagnóstico, identificando as variedades de tumor e detectando casos assintomáticos. ${ }^{8}$

\section{CASO CLÍNICO}

F.B.F. 39 anos, sexo feminino, aos 10 anos de idade apresentou uma crise descrita como repentina sensação de frio, seguido por turvação visual, perda de consciência e abalos clônicos generalizados, despertando depois com sensação de sede intensa que permaneceu por alguns minutos. Na época, por orientação de médico que a assistiu, iniciou uso de fenobarbital $100 \mathrm{mg}$ ao dia, mantido durante 4 anos, após tal período, estando assintomática, optou por suspender a medicação. Aos 22 anos de idade, durante gravidez, apresentou recidiva de crises, com a mesma semiologia, em frequência de cerca de duas crises por mês. Apesar disso, os médicos que a acompanharam na época optaram por não indicar reinício de medicação anti-epiléptica. Após a gravidez houve recrudescimento de crises, passando a manifestar aproximadamente 10 episódios por mês, assim persistindo até 38 anos de idade, em 2013, época em que foi avaliada por médico que prescreveu fenobarbital $100 \mathrm{mg}$ ao dia para tratamento anti-epiléptico. Desde então, experimentou redução de número de crises para dois episódios por mês.
Após alguns meses, por indicação de médico da estratégia de saúde da família, iniciou uso de carbamazepina $400 \mathrm{mg}$ ao dia, sem obter melhora. Aos antecedentes a paciente nega etilismo, tabagismo, uso de drogas ilícitas, alergias medicamentosas e demais doenças crônicas. Menciona ter realizado cirurgia prévia de colecistectomia por volta de 35 anos de idade.

Ao interrogatório sistemático a paciente referiu início de cefaleia desde 37 anos de idade, descritas como dor em região occipital bilateral, às vezes irradiada para região frontal, opressiva, de moderada intensidade, sem fotofobia, às vezes com fonofobia, não agravada por atividade física, sem náuseas, sem vômitos, negou piora catamenial ou piora ao se expor em demasia ao sol, relatou melhora com medicação analgésica, de duração de até 1 dia, com a cefaleia aumentanto em frequência com o decorrer do tempo, acontecendo atualmente numa frequência diária. Paciente afirma que vem sendo acompanhada por psiquiatra desde 2012 devido a ansiedade e depressão, referindo episódio de tentativa de suicídio em 2013, desde essa época fazendo uso de fluoxetina $20 \mathrm{mg}$ ao dia e diazepan $10 \mathrm{mg}$ ao dia, mantidos ainda nessas doses atualmente, ressaltando que houve melhora parcial de depressão de humor e importante melhora da cefaleia após o início das medicações.

$\mathrm{O}$ exame físico geral não evidenciou alterações relevantes. $\mathrm{O}$ exame neurológico demostrou funções corticais preservadas, fundoscopia e nervos cranianos normais, ausência de movimentos anormais, tônus e trofismo musculares normais, força muscular grau $5 \mathrm{em}$ todos os músculos pesquisados, reflexos osteotendíneos grau 2 obtidos em tendões bicipital, estilorradial, tricipital, patelar e aquileu bilateralmente, cutâneo-plantar flexor bilateralmente, sensibilidades preservadas, Rombergnegativo, normometria, eudiadococinesia e marcha normais. Exames complementares disponibilizados: ressonância magnética de crânio (Figura 1) concluída como imagem alongada, bem definida e com hipersinal em T1 e sem expressão em T2 e FLAIR, contornando o corpo caloso onde se estende do esplênio para o tronco, compatível com lipoma do corpo caloso do tipo curvilíneo. Tomografia de crânio realizada foi descrita como aparente malformação do corpo caloso.

Figura 1. Imagem de ressonância magnética de lipoma de corpo caloso (seta).

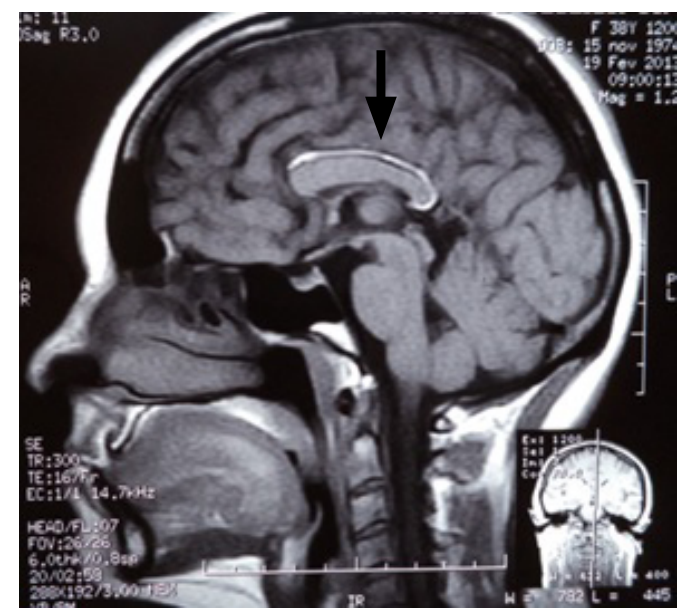




\section{DISCUSSÃO}

Os lipomas são descritos como raros defeitos da linha mediana, tendo em sua apresentação o acometimento de componentes intracerebral, extra-axial e extracraniano.

No caso clínico relatado, a paciente apresenta imagem compatível com lipoma intracraniano localizado no corpo caloso, localização mais habitual nesta situação clínica. De acordo com a literatura, List e col. ${ }^{9}$ e Kazner e col., ${ }^{1}$ descreveram os lipomas do sistema nervoso central mais frequentes no corpo caloso. Outras localizações descritas são: cisterna quadrigeminal (25\%), suprasselar/interpeduncular (14\%), ângulo pontocerebelar (9\%) e cisterna silviana (5\%). ${ }^{1,9}$ Kushnet e Goldman, ${ }^{10}$ relataram uma associação de aproximadamente $50 \%$ com agenesia do corpo caloso.

Quanto ao quadro clínico, a epilepsia é o achado mais comum no LCC, podendo ser parcial complexa ou motor parcial, sendo que essas crises podem evoluir para crises tônico-clônicas generalizadas. ${ }^{11}$ Outras formas de apresentação são cefaleias, vômitos, vertingens, labilidade emocional, retardo ou paralisia mental, quando existe acometimento, principalmente, do corpo caloso. ${ }^{12}$ Em 50\% dos casos, podem ser assintomáticos. A apresentação clínica tem relação com o acometimento das estruturas adjacentes com a lesão, assim como o comprometimento dos vasos e nervos cranianos e o sistema ventricular, estes tendo como manifestações hidrocefalia obstrutiva e pressão intracraniana elevada. ${ }^{13}$

A paciente não apresentava nenhuma anormalidade ao exame neurológico, mas apresentava crises convulsivas recorrentes ao longo da vida sem outras lesões encefálicas à exceção do LCC, manifestação que poderia ser decorrente deste. Apresentava também uma cefaleia do tipo tensional, com localização frontal, opressiva, de moderada intensidade, sem piora com atividade física, fotofobia, náuseas e vômitos,

\section{REFERÊNCIAS}

1. Kazner E, Stochdorph O, Sigurd W, Grumme T. Intracranial lipoma. J Neurosurg. 1980;52:234-45.

2. Silva DF, Lima MM, Oliveira CO, Oliveira WN, Anghinah R, Lima JG. Agenesis and lipoma of corpus callosum. Case report. Arq Neuropsiquiatr. 1995;53(3-B):667-70.

3. Martins RS, Orildo C Junior, Matushita H, Plese JP. Lipoma do corpo caloso com extensão extracraniana através de falha óssea frontal. Arq Neuropsiquiatr. 1995;53(3-B) 671-5.

4. Montandon C, Ribeiro FA, Lobo LV, Montandon Junior ME, Teixeira KI. Disgenesia do corpo caloso e más formações associadas: achados de tomografia computadorizada e ressonância magnética. Radiol Bras. 2003;36(5):311-6.

5. Pioversan EJ, Tatsui CE, Kowacs PA, Prazeres RF, Lange MC, Antoniuk SA, et al. Lipoma do corpo caloso associado a hipertrofia do corpo caloso. Arq Neuropsiquiatria. 2000;58(3B):947-51.

6. Zubair A, Zehra N, Masood J, Ullah S, Khattak JZ. Epilepsy: melhora com medicação analgésica, de duração de até 1 dia, e melhora após o início de fluoxetina e benzodiazepínico.

Na Tomografia de crânio (TC), o LCC é hipoatenuante, sem realce com contraste, bem delimitado e sem edema próximo à lesão. Quanto à presença de calcificações, estas podem ser visualizadas na periferia, envolta de uma cápsula fibrosa, padrão característico do LCC. ${ }^{12}$ A RNM é o exame de escolha, pois fornece imagens do corpo caloso com boa visualização. Quanto ao diagnóstico diferencial temos os cistos dermoides e os teratomas, que na TC, podem ter semelhança na imagem. Essas patologias apresentam-se com mais frequência nas regiões subfrontais, subtemporal e terceiro ventrículo. São imagens heterogêneas, diferente dos lipomas que são homogêneos. ${ }^{14}$

Quanto ao tratamento, a indicação de procedimento cirúrgico tem pouco benefício nesta patologia, visto que se trata de uma lesão benigna, sem caráter progressivo e de bom prognóstico, podendo o indivíduo chegar, sem complicações, a senilidade. Segundo Kazner e col., os pacientes submetidos a cirurgia não apresentam remissão das crises convulsivas, sendo esta a manifestação mais comum. ${ }^{1}$

Nesta paciente, foi definido conduta com tratamento clínico com medicamentos anticonvulsivantes e seguimento ambulatorial, evoluindo com controle das crises convulsivas e sem outras manifestações.

\section{CONSIDERAÇÕES FINAIS}

O lipoma de corpo caloso é uma malformação benigna e rara. A utilização de métodos de análise por neuroimagem permitiu o diagnóstico, visto que boa parte dos pacientes são assintomáticos. Os avanços dos métodos de identificação por imagem proporcionaram um aumento de detecção. Seu tratamento, a princípio, é conservador, e em casos selecionados pode-se optar por tratamento cirúrgico.

neurological disorder-a review. Asian J Med Sci. 2014;6(3):25-9.

7. Internacional League Against Epilepsy. Epilepsies by ttiology [Internet]. Flower Mound: Internacional League Against Epilepsy; [acesso em: 22 Jan 2015]. Disponível em: https://www. epilepsydiagnosis.org/aetiology/epilepsies-etiology-groupoverview. html

8. Robins ST, Cotran RS, Kumar V. Aspectos clínicos das neoplasias. In: Andrade ZA, Barreto M. Patologia estrutural e funcional. 3. ed. Rio de Janeiro: Guanabara SA; 1986. p. 263-4.

9. Lits CF, Holt JF, Everett M. Lipoma of the corpus callosum: a clinicopathologic study. Am J Roentgenol Radium Ther Nucl Med. 1946;55:125-34.

10. Kushnet MW, Goldman RL. Lipoma of corpus callosum associated with a frontal bone defect. Am J Roentgenol. 1978;131:517-8.

11. Gastaut H, Regis H, Gastaut JL, Yermenos E, Low MD. Lipomas of the corpus callosum and epilepsy. Neurology. 1980;30:132-8. 
12. Pereira CU, Silveira AC, Barreto AS, Britto AV, Barbosa BJ. Lipoma intracraniano - revisão de literatura. Arq Bras Neurocir. 2013;32(2):98-104.

13. Zettner A, Netsky M. Lipoma of the corpus callosum. J Neuropathol Exp Neurol. 1960;19:305-19.
14. NEW PF, SCOTT WR. Computed tomography of the brain and orbit (EMI-Scanning). Baltimore: Williams \& Wilkins; 1975.

\section{Como citar:}

Amorim FD, Macedo IO, Leite MP Neto, Cunha FM, Romualdo IM. Lipoma de corpo caloso: relato de caso. Rev Med UFC. 2018 out-dez;58(4):79-82. 